\title{
Mutual Coupling in Antenna Arrays 2011
}

\author{
Hoi Shun Lui, ${ }^{1}$ Hon Tat Hui, ${ }^{2}$ and Marek Bialkowski ${ }^{3}$ \\ ${ }^{1}$ Department of Signals and Systems, Chalmers University of Technology, 41296 Gothenburg, Sweden \\ ${ }^{2}$ Department of Electrical and Computer Engineering, National University of Singapore, 4 Engineering Drive 3, Singapore 117576 \\ ${ }^{3}$ School of Information Technology and Electrical Engineering, University of Queensland, St Lucia, Brisbane, QLD 4072, Australia \\ Correspondence should be addressed to Hoi Shun Lui, antony.lui@chalmers.se
}

Received 16 February 2012; Accepted 16 February 2012

Copyright (C) 2012 Hoi Shun Lui et al. This is an open access article distributed under the Creative Commons Attribution License, which permits unrestricted use, distribution, and reproduction in any medium, provided the original work is properly cited.

Mutual coupling in antenna arrays is a topic of continuous interest to the researchers and application engineers in the field. Since the application of antenna arrays has been extended to many areas in modern days, the study of the mutual coupling phenomenon has become a popular and important topic of research. We have seen many new methods to analyse compensate for the mutual coupling effect in the last few years. In 2009, we have coordinated the first special issue on "Mutual Coupling in Antenna Arrays". We have received 16 submissions and 9 of them have been recommended for publication. In this second special issue, we have received 29 manuscripts, out of which 17 have been recommended for publication by dedicated reviewers. All the accepted papers in both special issues have been subject to a strict peer-reviewing process. On one hand, we greatly acknowledge the reviewers for their professional reviews of the manuscripts, while, on the other hand, we are pleased to see that a much larger attention has been paid by the authors to this second special issue. The 17 accepted manuscripts can be grouped into five categories: the implication of mutual coupling problem in antenna array design, mutual coupling in MIMO system antenna arrays, mutual coupling in direction finding problems, mutual coupling in wideband antenna arrays, and calibration methods for the mutual coupling effect. A quick reference to these papers is given below to introduce them to the interested readers.

In the first category, the paper "An optimal design of multiple antenna positions on mobile devices based on mutual coupling analysis" by P. Uthansakul et al. introduces a method of using the genetic algorithm (GA) to find the optimal antenna positions on a mobile device in order to achieve an optimum operation under the influence of the mutual coupling effect. In the paper, "Synthesis of antenna arrays and parasitic antenna arrays with mutual couplings" by M. Thevenot et al., the authors present a synthesis method for various types of moderate-size antenna arrays together with their feeding networks with an aim to optimize the array performance in the presence of mutual coupling effect. The paper "A Compact low-permittivity dual-layer ebg structure for mutual coupling reduction" by A. Azarbar and J. Ghalibafan proposes a dual layer electromagnetic bandgap (EBG) structure, which has a lower resonant frequency than a single-layer one, to reduce the mutual coupling between an E-plane-coupled microstrip antenna array, resulting in a significant $19 \mathrm{~dB}$ mutual coupling reduction. The paper "Mutual coupling effects analysis in a cross-rhombic antenna array" by J. Sosa-Pedroza et al. presents a full-wave simulation analysis of the mutual coupling effect in a conformal array consisted of cross-rhombic antennas. Results have been obtained on the radiation patterns and the S11 parameters of the array. The paper "A novel design of microstrip arrays for relay-based wireless network" by I. Petropoulos et al. reports a design of relay-station antenna arrays based on reducing the mutual coupling between the access link array and the backhaul link array, achieving a coupling typically less than $-40 \mathrm{~dB}$. In the last paper of this category, "Optimization of a $12.5 \mathrm{GHz}$ microstrip antenna array using Taguchi's method" by M. Spasos, et al., the authors present an optimization design of a simple planar $5 \times 5$ microstrip antenna array with interconnected elements to achieve a good input matching (with $\mathrm{S} 11<-10 \mathrm{~dB}$ ) without using any matching network. They used the Taguchi's optimization method in the design which shows low interelement mutual coupling as well as good self-impedance matching.

In the second category, the paper "Investigation into the impact of mutual coupling on the performance of a multiuser 
mimo system employing generalized channel inversion technique" by F. Wang et al. reports an investigation into the performance of a multiuser MIMO system employing generalized channel inversion scheme. The investigation specially considers the effects of spatial correlation and antenna mutual coupling which are significant in compact array antennas. In the paper "An envelope correlation formula for $(N, N)$ MIMO antenna arrays using input scattering parameters, and including power losses" by Y. A. S. Dama et al., the authors present a method to derive a closed-form expression for the correlation coefficient in terms of the scattering parameters of an antenna array. This expression takes into account the antenna mutual coupling as well as possible antenna losses into account. The paper "Optimal antenna selection designs for coupled MIMO systems" by $\mathrm{P}$. S. Taluja and B. L. Hughes reports that the presence of inactive antennas in a coupled MIMO system can profoundly alter the system performance when the antennas are strongly coupled. A new antenna selection technique that seeks to exploit mutual coupling to improve the system performance is proposed. In the last paper in this category, "The noise performance of a multiple-input-port and multiple-output-port low-noise amplifier connected to an array of coupled antennas" by F. Broydé and E. Clavelier, the authors investigated a noise characterization method for low-noise amplifiers connected to a mutually coupled antenna arrays. The noise figures of the low-noise amplifiers were defined and determined.

In the third category, the paper "Spatial correlation for doa characterization using von mises, cosine, and gaussian distributions" by W. J. L. Queiroz et al. presents mathematical expressions for the spatial correlation between elements of linear and circular antenna arrays, considering cosine, Gaussian, and Von Mises distributions for the direction-of-arrival (DoA) of the electromagnetic waves at the receiver antenna. The expressions obtained for the Von Mises distribution can include or exclude the mutual coupling effect and are simpler than those obtained for the cosine and the Gaussian distributions of the angle of arrival. In the paper "Effects of ground constituent parameters on array mutual coupling for DOA estimation" by I. Ahmed et al., the authors suggested the use of an imperfect ground plane to reduce the mutual coupling effect of a monopole antenna array mounted over the imperfect ground plane. A factor of 4 in the decrease in both the real and imaginary parts of the mutual coupling effect is reported. In the paper "A Subspace-based compensation method for the mutual coupling in concentric circular ring arrays for near-field source localisation" by M. J. Abedin and A. S. Mohan, the authors proposed a technique for compensating the effect of mutual coupling on parameter estimation that is suitable with any subspacebased superresolution algorithms. The method has been demonstrated on a concentric circular-ring array formed by thin-dipole antennas in the receiving mode for near-field source localization.

In the fourth category, there are two papers. The first paper "Wideband analysis of mutual coupling compensation methods" by S. Henault and Y. M. M. Antar compares the performance of various mutual coupling estimation and compensation methods for wideband antenna arrays. The main limitations and the required procedures to estimate the coupling matrix are outlined. This paper found that only the full-wave method is accurate at all frequencies. In the second paper "Mutual coupling effect on ultrawideband antenna array performance" by O. Ahmed and A. R. Sebak, the mutual coupling effect between array elements of twoand four-element ultrawideband (UWB) linear arrays on the performances of these arrays is investigated. This study found that an array bandwidth improvement is achieved for both array types when mutual coupling is strong enough. It also found that mutual coupling enhances the array realizable gain, especially in the mid-frequency band $(5-8.5 \mathrm{GHz})$ while it deteriorates the gain outside this frequency range.

In the last category, the first paper "A measurement system for the position and phase errors of the elements in an antenna array subject to mutual coupling" by T. Lindgren and J. Borg discusses a method of estimating the position and phase of each individual antenna element in an array in the presence of mutual coupling. The method uses both the measured scattering matrix and the measured electric field with four probes located in the near-field of the array. The second paper "An external calibration method for compensating for the mutual coupling effect in large interferometric aperture synthesis radiometers" by J. Dong deals with the antenna mutual coupling effect in interferometric aperture synthesis radiometers (IASRs) which degrades the radiometric system performance. First, the conventional mutual impedance method is adopted to analyze the mutual coupling effect in the performance of IASR and a practical model of the coupled visibilities is developed. Based on the model, an external calibration method is proposed to compensate for the mutual coupling effect.

The papers in this second special issue came from various types of studies. These papers reported the handling of the mutual coupling problem in antenna arrays for various applications. Through the collection of these papers, we hope to bring out the state-of-the-art research on mutual coupling in antenna arrays such that more ideas and research on this topic can be encouraged to come out in the future.

Hoi Shun Lui

Hon Tat Hui

Marek Bialkowski 

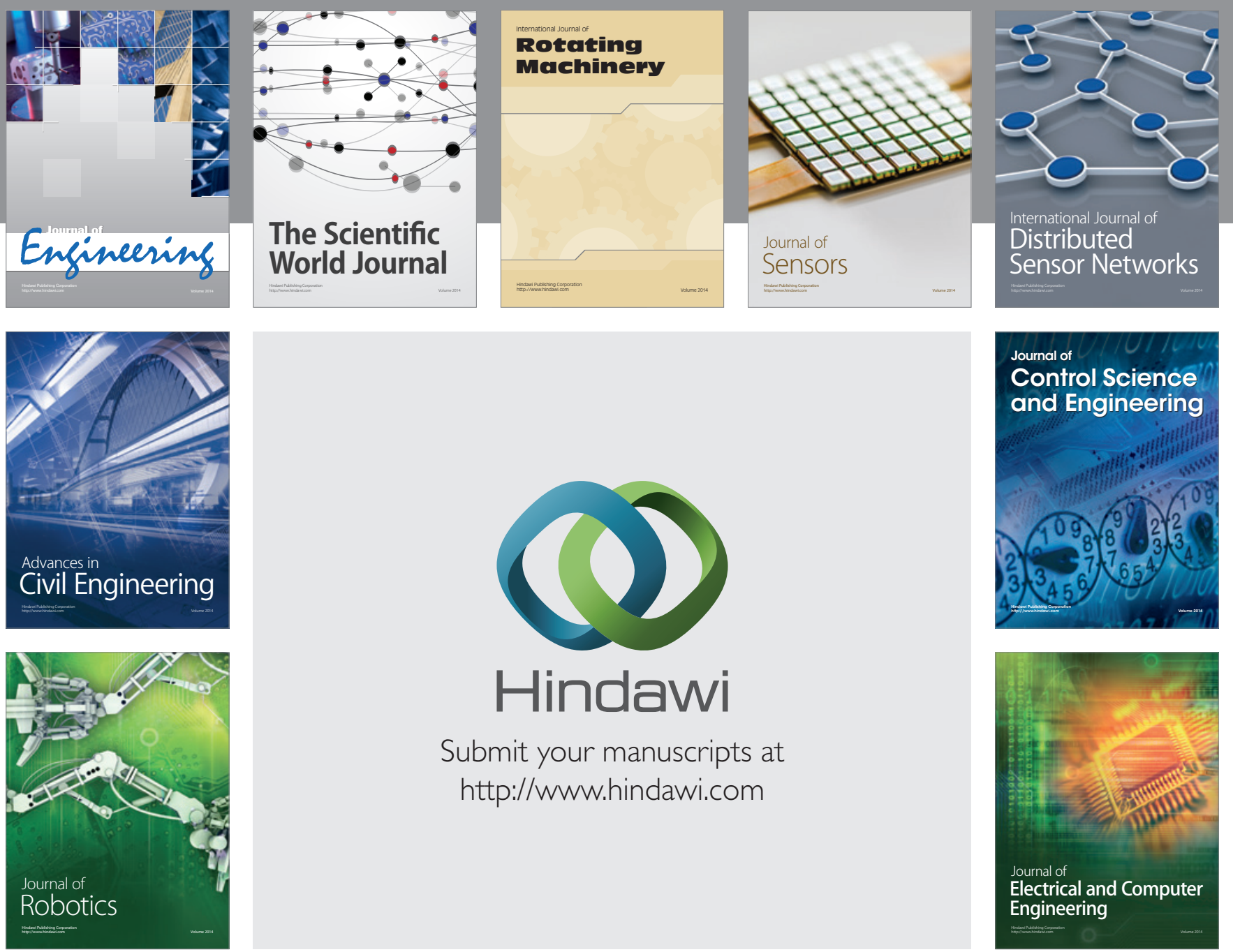

Submit your manuscripts at

http://www.hindawi.com
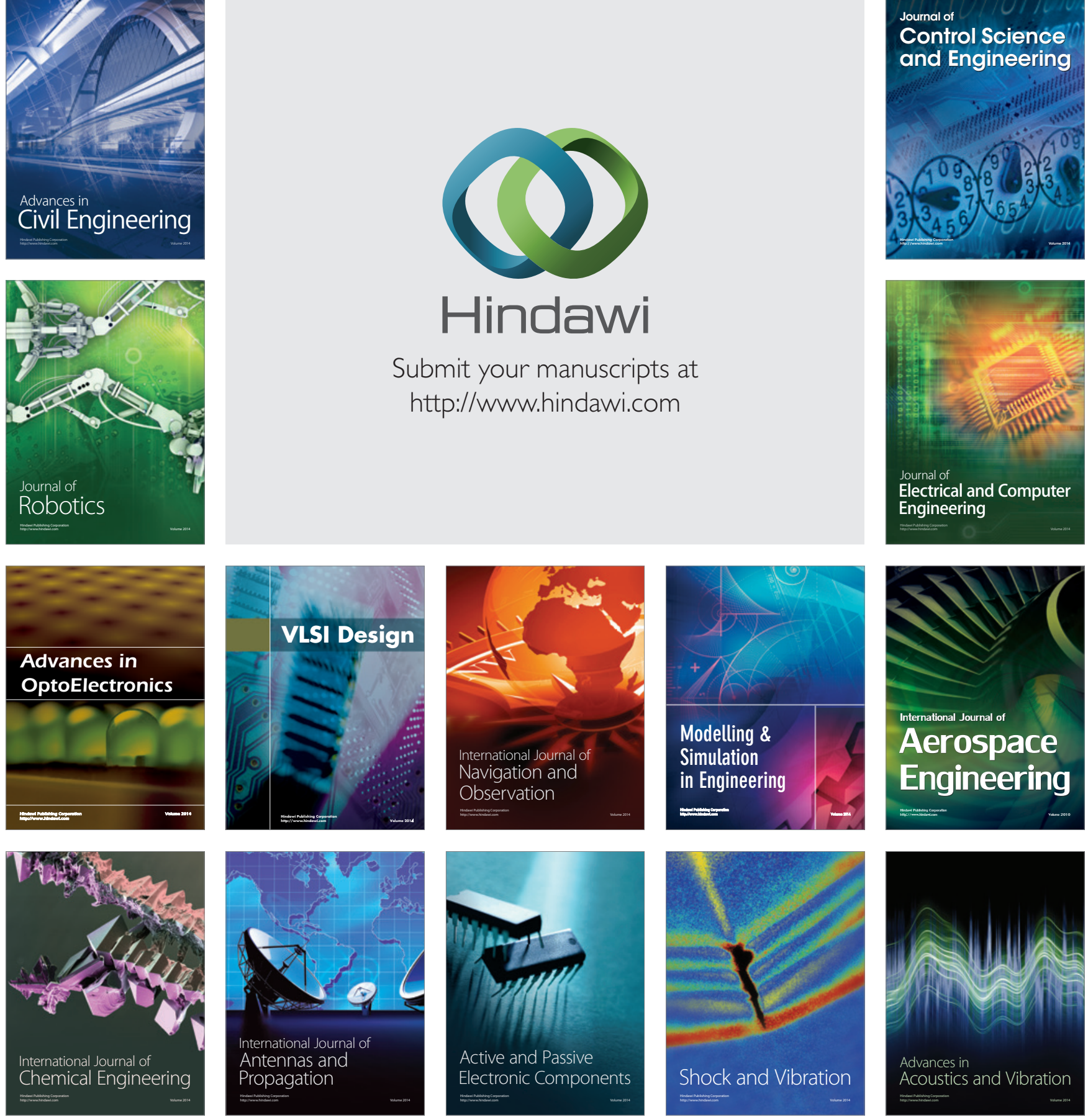